\title{
Benign and malignant pulmonary part-solid nodules: differentiation via thin-section computed tomography
}

\author{
Wang-Jia Li ${ }^{1 \# \wedge}$, Fa-Jin $\mathrm{Lv}^{1 \#}$, Yi-Wen Tan ${ }^{2}$, Bin-Jie Fu ${ }^{1 \wedge}$, Zhi-Gang Chu ${ }^{1} \wedge$ \\ ${ }^{1}$ Department of Radiology, The First Affiliated Hospital of Chongqing Medical University, Chongqing, China; ${ }^{2}$ Department of Pathology, The First \\ Affiliated Hospital of Chongqing Medical University, Chongqing, China
}

Contributions: (I) Conception and design: ZG Chu; (II) Administrative support: FJ Lv; (III) Provision of study materials or patients: ZG Chu, FJ Lv; (IV) Collection and assembly of data: WJ Li, FJ Lv; (V) Data analysis and interpretation: YW Tan, BJ Fu; (VI) Manuscript writing: All authors; (VII) Final approval of manuscript: All authors.

\#These authors contributed equally to this work and should be considered as co-first authors.

Correspondence to: Zhi-Gang Chu, MD, PhD. Department of Radiology, The First Affiliated Hospital of Chongqing Medical University, 1\# Youyi Road, Yuanjiagang, Yuzhong District, Chongqing 400016, China. Email: chuzg0815@163.com.

Background: Pulmonary part-solid nodules (PSNs) reportedly have a high possibility of malignancy, while benign PSNs are common. This study aimed to reveal the differences between benign and malignant PSNs by comparing their thin-section computed tomography (CT) features.

Methods: Patients with PSNs confirmed by postoperative pathological examination or follow-up (at the same period) were retrospectively enrolled from March 2016 to January 2020. The clinical data of patients and CT features of benign and malignant PSNs were reviewed and compared. Binary logistic regression analysis was performed to reveal the predictors of malignant PSNs.

Results: A total of 119 PSNs in 117 patients [age (mean \pm standard deviation), $56 \pm 11$ years; 70 women] were evaluated. Of the 119 PSNs, 44 (37.0\%) were benign, and 75 (63.0\%) were malignant (12 adenocarcinomas in situ, 22 minimally invasive adenocarcinomas, and 41 invasive adenocarcinomas). There were significant differences in the patients' age and smoking history between benign and malignant PSNs. In terms of CT characteristics, malignant and benign lesions significantly differed in the following CT features: whole nodule, internal solid component, and peripheral ground-glass opacity. The binary logistic regression analysis revealed that well-defined border [odds ratio (OR), 4.574; 95\% confidence interval (CI), 1.186-17.643; $\mathrm{P}=0.027$ ] and lobulation (OR, 61.739; 95\% CI, 5.230-728.860; $\mathrm{P}=0.001$ ) of the nodule, as well as irregular shape (OR, 9.502; 95\% CI, 1.788-50.482; $\mathrm{P}=0.008)$ and scattered distribution (OR, 13.238; 95\% CI, 1.359-128.924; $\mathrm{P}=0.026$ ) of the internal solid components were significant independent predictors distinguishing malignant PSNs. However, the lesion shape, density, and margin were similar between malignant and benign lesions.

Conclusions: Well-defined and lobulated PSNs with irregular and scattered solid components are highly likely to be malignant.

Keywords: Part-solid nodules (PSNs); computed tomography (CT); malignancy; benignity; solid component

Submitted Feb 05, 2021. Accepted for publication Aug 11, 2021.

doi: $10.21037 /$ qims-21-145

View this article at: https://dx.doi.org/10.21037/qims-21-145

\footnotetext{
^ ORCID: Zhi-Gang Chu, 0000-0002-2441-8132; Wang-Jia Li, 0000-0002-8633-0101; Fa-Jin Lv, 0000-0002-6484-9738; Bing-Jie Fu, $0000-0002-6863-2827$.
} 


\section{Introduction}

With the widespread application of computed tomography (CT) screening for lung cancer, the increasing detection of ground-glass nodules (GGNs) has attracted attention owing to their high possibility of being early lung cancers (1-4). GGNs can be further classified into pure GGNs and part-solid nodules (PSNs) based on the absence or presence of a solid component (5). PSNs have been reported to be associated with lung adenocarcinoma, and their malignancy rate is higher than that of pure GGNs and solid nodules $(6,7)$. Pathologically, PSNs are mainly confirmed as minimally invasive and invasive adenocarcinomas (IAs), while pure GGNs are mostly adenocarcinomas in situ and sometimes minimally invasive adenocarcinomas (MIAs) (8). Therefore, an aggressive approach to treatment is usually recommended for PSNs, especially for lesions with solid components larger than $5 \mathrm{~mm}$ (5).

However, PSN is a nonspecific radiological manifestation, which could also be found in various benign conditions, such as inflammatory disease, focal interstitial fibrosis, or alveolar hemorrhage $(9,10)$. Furthermore, some PSNs could be spontaneously resolved during follow-up. Oh et al. (11) found that a significant portion (48.7\%) of PSNs disappeared over follow-up, and most of them were resolved within 3 months after initial detection. Therefore, many PSNs could be benign lesions (BLs), and it is important to differentiate BLs from malignant lesions (MLs) based on initial CT data for further management of PSNs.

Previous studies have investigated the differences in CT features of PSNs with different natures (11-13). Yang et al. (12) reported that large size, well-defined border, and spiculation favored the diagnosis of MLs, and Lee et al. (13) revealed that lesion multiplicity, large solid component, and ill-defined lesion border indicated transient lesions. Although the differences in size and CT value of the internal solid component have also been investigated (12-14), these studies mainly compared the overall CT features of nodules rather than comprehensively analyzing the differences between their various components. Moreover, some results were acquired based on the enrolled cases confirmed only by follow-up, which limited the clinical value of their findings. The current study enrolled patients with benign PSNs confirmed by follow-up or pathology and MLs by pathology, and the CT features of the whole nodule, internal solid component, and peripheral GGO component were analyzed and compared, respectively. These findings may provide more information for differentiating benign and malignant PSNs.

\section{Methods}

This retrospective study used anonymized patient information for data analysis and was conducted in accordance with the Declaration of Helsinki (as revised in 2013). The ethics committee approved this study of the First Affiliated Hospital of Chongqing Medical University, and the requirement for written informed consent was waived due to its retrospective nature.

\section{Study population}

From March 2016 to January 2020, patients with pathologically confirmed benign GGNs or resolved GGNs were collected, and cases with pathologically confirmed malignant GGNs during the same period were also collected. Cases that met the following criteria were subsequently enrolled in this study: (I) patients with pulmonary GGNs manifesting as PSNs; and (II) patients' clinical data were complete. PSN was defined as a nodule manifesting as a GGO (that does not completely obscure the underlying lung parenchyma within them) with a solid component (that obscures the underlying lung parenchyma other than blood vessels on lung window setting) on thin-section CT. The exclusion criteria were as follows: (I) CT images with a thickness $>1 \mathrm{~mm}$; and (II) presence of artifacts on CT images affecting evaluation. Finally, 117 patients with 119 PSNs were included in this study. The patients' selection procedure is shown in Figure 1.

\section{CT examinations}

All patients underwent non-contrast chest CT with a 128-slice multi-detector CT scanner (SOMATOM Definition Flash system, Siemens Medical Systems, Germany) while holding their breath after inspiration, and were scanned from thoracic inlet to the lung base. CT examinations were performed with the following parameters: tube voltage, $110-120 \mathrm{kV}$; tube current, 50-150 mAs; beam pitch, 1.0; detector collimation, $0.6 \mathrm{~mm}$; rotation time, $0.5 \mathrm{~s}$; reconstruction thickness, 1.0 or $0.625 \mathrm{~mm}$; reconstruction interval, 1.0 or $0.625 \mathrm{~mm}$; and reconstruction kernel, medium-sharp algorithm. 


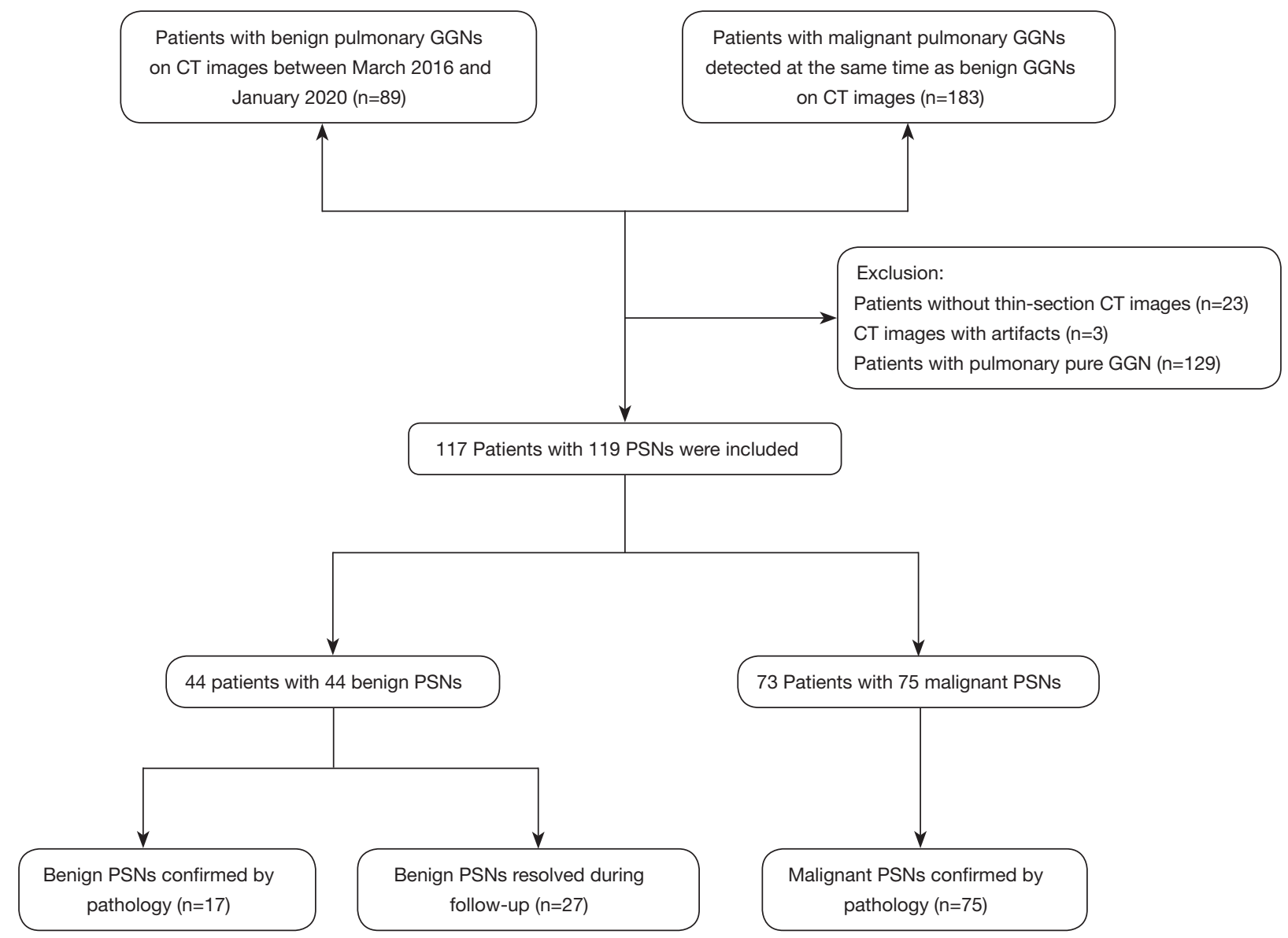

Figure 1 Flowchart of study population. CT, computed tomography; GGN, ground-glass nodule; PSN, part-solid nodule.

\section{Analysis of CT features}

CT images of all patients were reviewed by two radiologists (with more than 10 years of experience in chest CT interpretation) who were blinded to the clinical data and pathological results. Any disagreements between the two radiologists during the evaluation were resolved by consensus. CT images were analyzed with lung window setting (window level, -600 HU; width, 1,600 HU) using Picture Archiving and Communication System (PACS, Carestream, USA) and multiplanar reconstruction.

The overall CT features of each PSN were evaluated: (I) lesion size (mean of the longest diameter and the perpendicular diameter on axial images); (II) lesion area (the largest area of the entire PSN on axial images); (III) location; (IV) lesion shape (round, oval, or irregular); (V) lesion border (well-defined or ill-defined); (VI) lesion margin (smooth or coarse); (VII) lobulation (a portion of the lesion's surface showing wavy or scalloped configuration); (VIII) spiculation (linear strands extending beyond the lesion); (IX) air bronchogram (visible air-filled bronchi detected in the nodule); $(\mathrm{X})$ bubble lucency (small spots of air attenuation within the lesion); (XI) pleural indentation (pulling of the visceral pleura towards the pulmonary nodule), and (XII) pulmonary vessel changes (distorted, dilated, or both). For the GGO component, its density and uniformity (homogeneous or heterogeneous) were also evaluated.

The CT features that were analyzed for solid component included the following: (I) area (the largest area of the internal solid component on axial images); (II) solid ratio (the largest area of the solid component divided by largest lesion area); (III) density; (IV) number (solitary or multiple); (V) shape (round, oval, or irregular); (VI) border (well-defined or ill-defined); (VII) margin of well-defined 
solid component (smooth or coarse); (VIII) distribution (concentrated or scattered), and (IX) location (central, eccentric).

The border was considered ill-defined when the interface between the lesion and peripheral lung tissue was totally or partly unclear; otherwise, it was considered well-defined. Similarly, the margin was considered coarse when any lesion surface was unsmooth; otherwise, it was considered smooth. We did not record the size for multiple and irregular solid components to preclude reliable and accurate evaluation. The CT attenuation of solid components was measured by placing multiple regions of interest (ROIs) (multiple solid components) or at least three ROIs on different sections (single solid component). The ROIs covered two-thirds of the largest area and avoided internal vessels. The CT attenuation of GGO components was measured by placing at least three ROIs in different areas. The average of multiple measurements for solid components and GGO was the final results.

\section{Clinical and pathological data}

The patients' clinical and laboratory data were recorded using the Electronic Medical Record System (Winning Health, China). Clinical data, including the patient's age, sex, smoking history (never-smoker, ex-smoker, or current smoker), smoking amount, and history of cancer, were recorded. Laboratory findings, such as white blood cell (WBC) count, blood eosinophil count, and presence of blood eosinophilia, were also recorded. These laboratory examinations were performed preoperatively and within 1 week after CT examination.

Two pathologists reviewed all existing histopathological slides, and histopathological analysis was performed according to the 2015 World Health Organization classification of tumors of the lung, pleura, thymus, and heart (15).

\section{Statistical analysis}

Continuous data (patients' age, smoking amount, WBC count, and blood eosinophil count; lesions' size and area; density of GGO; solid components' area, ratio, and density) were expressed as mean \pm standard deviation or median (range), whereas categorical variables were presented as numbers and percentages. Continuous data were analyzed using the analysis of variance (patients' age, lesion size, and density of GGO) or Wilcoxon rank-sum test (patients' smoking amount, lesion area, solid component area, solid component ratio, and density). Categorical data were analyzed by Pearson's $\chi^{2}$ test (patients' sex, lesion shape and border, lobulation, spiculation, air bronchogram, bubble lucency, pleural indentation, pulmonary vessel changes, uniformity of GGO, solid component number, shape, border distribution, and location) or Fisher's exact test (patients' smoking history, history of cancer, WBC count, blood eosinophil count, blood eosinophilia, lesion location, and margin of well-defined border). A P value less than 0.05 was considered to indicate a statistically significant difference. All statistical analyses were performed using SPSS 20.0 (SPSS, Chicago, IL, USA).

Binary logistic regression analysis was performed to identify variables that could be used to differentiate benign from malignant PSNs. Due to multi-collinearity in some clinical data and CT features, the least absolute shrinkage and selection operator (LASSO) was used to further select features. Characteristics with a $\mathrm{P}$ value $<0.05$ in the univariate analysis were used as the independent variables for LASSO analysis, and the selected variables were then included in the binary logistic regression analysis. Receiver operating characteristic (ROC) analyses were conducted for lesion size and variables with statistically significant differences in the logistic regression analysis.

\section{Results}

\section{Clinical features}

In total, 117 patients (mean age, 56 \pm 11 years; range, 24-79 years) were enrolled in this study, including $70(59.8 \%)$ women (mean age, $56 \pm 11$ years; range, $29-84$ years) and $47(40.2 \%)$ men (mean age, $55 \pm 11$ years; range, $25-76$ years). Thirty-one $(31 / 117,26.5 \%)$ patients had a history of smoking with a median of 26.0 pack-years (range, $0.8-80.0$ pack-years), and $10(8.5 \%)$ patients had a history of cancer. Only two patients had blood eosinophilia and were diagnosed with benign nodules.

The clinical data of the 117 patients with BLs and MLs are summarized in Table 1. Among the 117 patients with 119 PSNs, there were 73 (62.4\%) patients with 75 (63.0\%) MLs, including 12 (16.0\%) adenocarcinomas in situ (AISs), $22(29.3 \%)$ MIAs, and 41 (54.7\%) IAs. Of the 44 (37.6\%) 
Table 1 Clinical features of 117 patients

\begin{tabular}{|c|c|c|c|}
\hline Clinical features & Patients with benign PSNs $(n=44)$ & Patients with malignant PSNs $(n=73)$ & $P$ value \\
\hline Sex & & & $0.196^{* *}$ \\
\hline Male & $21(47.7)$ & $26(35.6)$ & \\
\hline Female & $23(52.3)$ & $47(64.4)$ & \\
\hline Never-smoker & $25(56.8)$ & $61(83.6)$ & \\
\hline Ex-smoker & $5(11.4)$ & $3(4.1)$ & \\
\hline Current smoker & $14(31.8)$ & $9(12.3)$ & \\
\hline Smoking amount (pack-years) & $20.0(2.5-80.0)$ & $30.0(0.8-60.0)$ & $0.404^{\dagger}$ \\
\hline Absent & $42(95.5)$ & $65(89.0)$ & \\
\hline WBC count $(/ \mu \mathrm{L})$ & $5,875(3,130-14,520)$ & $5,490(1,026-17,230)$ & $0.051^{\dagger}$ \\
\hline Blood eosinophil count $(/ \mu \mathrm{L})$ & $75(0-1,130)$ & $65(10-440)$ & $0.930^{\dagger}$ \\
\hline Blood eosinophilia & & & $0.139^{\star \star \star}$ \\
\hline$\geq 500$ per microliter & $2(4.5)$ & $0(0.0)$ & \\
\hline$<500$ per microliter & $42(95.5)$ & $73(100.0)$ & \\
\hline
\end{tabular}

Data are presented as $\mathrm{n}(\%)$, means \pm SD or median (range). * , independent samples $t$-test; ${ }^{* *}$, calculated with the Pearson $\chi^{2}$ test; ${ }^{\star \star \star}$, calculated with the Fisher exact test; ${ }^{\dagger}$, Mann-Whitney U test. PSN, part-solid nodule; SD, standard deviation; WBC, white blood cell.

patients with 44 (37.0\%) BLs, 17 (38.6\%) were completely resolved during follow-up, and 27 (61.4\%) were confirmed by postoperative pathologic examination. Patients with MLs were older than patients with $\mathrm{BLs}(\mathrm{P}=0.013)$, and most of them were non-smokers $(\mathrm{P}=0.005)$.

\section{CT features of benign and malignant PSNs}

The overall CT findings of benign and malignant PSNs are shown in Table 2. The MLs were significantly larger than the BLs $(\mathrm{P}<0.001)$ and were more likely to have a well-defined border $(\mathrm{P}<0.001)$ and heterogeneous GGO $(\mathrm{P}=0.004)$. The optimal cut-off value to differentiate MLs from BLs was $12.5 \mathrm{~mm}$, with an area under the curve (AUC) of 0.734 [95\% confidence interval (CI), $0.642-0.826$, sensitivity and specificity: $73.3 \%$ and $63.6 \%$, respectively]. As for the morphological features, the MLs showed lobulation $(\mathrm{P}<0.001)$, bubble lucency $(\mathrm{P}=0.001)$, and pleural indentation $(\mathrm{P}<0.001)$ more frequently than the BLs (Figure 2). Also, all PSNs with spiculation, air bronchogram, and pulmonary vessel changes were MLs.

The CT findings of solid components in BLs and MLs are summarized in Table 3. The solid components in MLs were significantly larger than those in $\mathrm{BLs}(\mathrm{P}<0.001)$ and were more likely to be multiple $(\mathrm{P}<0.001)$, irregular $(\mathrm{P}<0.001)$, scattered $(\mathrm{P}<0.001)$, and eccentric $(\mathrm{P}=0.008)$ (Figures 2,3). Meanwhile, the solid components in BLs were usually single, round, and central (Figure 4) and were more likely to be ill-defined $(\mathrm{P}<0.001)$.

Binary logistic regression analysis revealed that welldefined border [odds ratio (OR), 4.574; 95\% CI, 1.18617.643; $\mathrm{P}=0.027]$ and lobulation $(\mathrm{OR}, 61.739 ; 95 \% \mathrm{CI}$, 5.230-728.860; $\mathrm{P}<0.001)$ of the lesion, as well as irregular shape (OR, 9.502; 95\% CI, 1.788-50.482; $\mathrm{P}=0.008)$ and scattered distribution (OR, 13.238; 95\% CI, 1.359-128.924; $\mathrm{P}=0.026)$ of the solid components were significant 
Table 2 The overall CT findings of benign and malignant PSNs

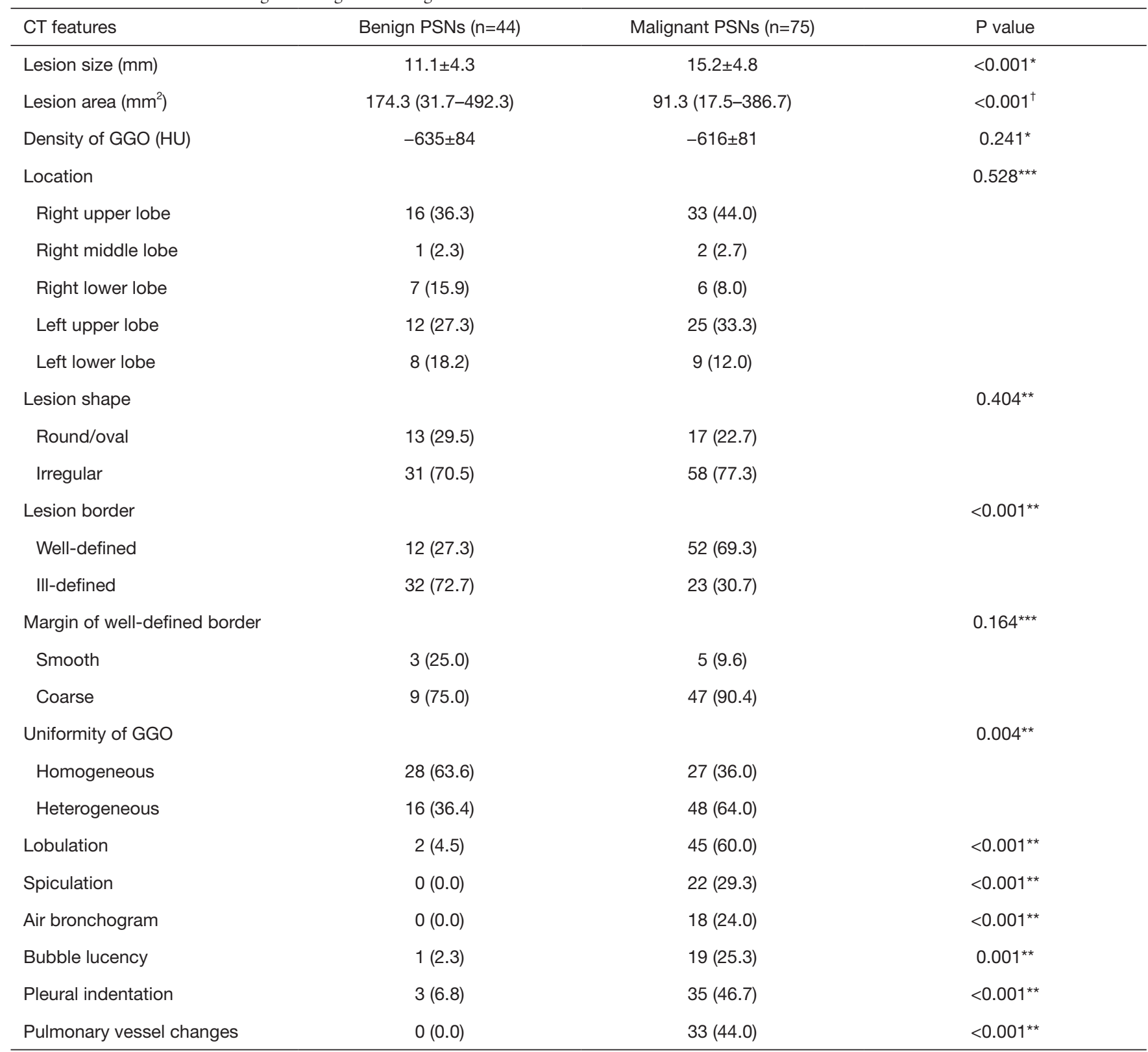

Data are presented as $\mathrm{n}(\%)$, means \pm SD or median (range). *, independent samples t-test; ${ }^{\star *}$, calculated with the Pearson $\chi^{2}$ test; ${ }^{\star \star \star}$, calculated with the Fisher exact test; ${ }^{\dagger}$, Mann-Whitney $U$ test. CT, computed tomography; GGO, ground-glass opacity; SD, standard deviation; PSN, part-solid nodule.

predictors distinguishing malignant PSNs. ROC analysis revealed that the AUCs for the border and lobulation of the nodule, and the shape and distribution of the solid components were 0.710 (95\% CI, 0.613-0.808; $\mathrm{P}<0.001$ ), 0.775 (95\% CI, 0.691-0.858; $\mathrm{P}<0.001$ ), 0.737 (95\% CI, $0.638-0.837 ; \mathrm{P}<0.001$ ), and 0.731 (95\% CI, 0.642-0.820; 

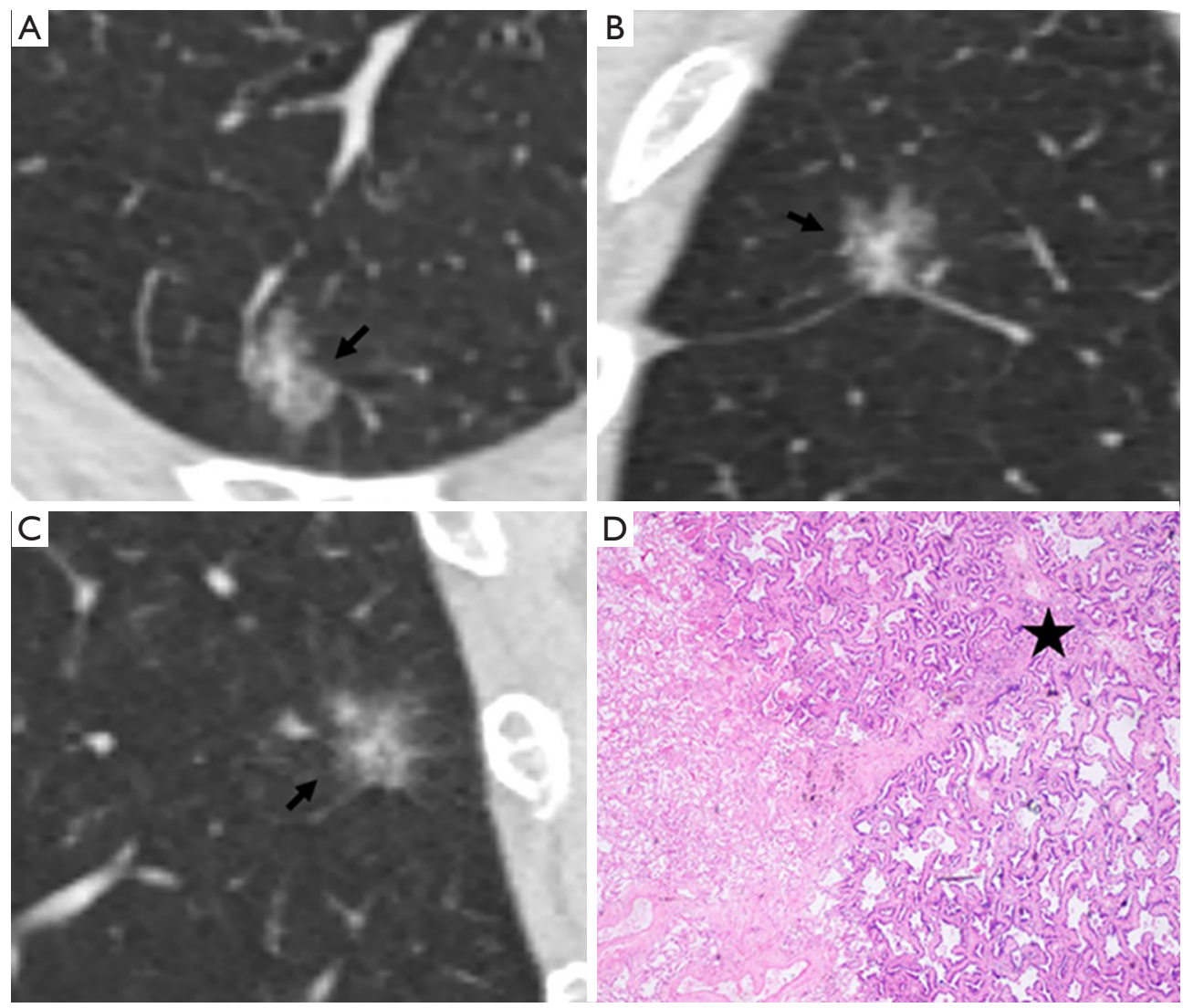

Figure 2 A 55-year-old woman with an invasive adenocarcinoma. (A-C) Axial (A), coronal (B), and sagittal (C) CT images show a 13-mm lobulated and well-defined PSN (arrows) in the right upper lobe. The internal solid components are multiple, scattered, and irregular, and the peripheral GGO is heterogeneous. (D) Photomicrograph (hematoxylin-eosin stain, $\times 100$ ) of the PSN reveals adenocarcinoma with invasive foci (asterisk). CT, computed tomography; GGO, ground-glass opacity; PSN, part-solid nodule.

$\mathrm{P}<0.001$ ), respectively (Figure 5).

\section{Discussion}

Most PSNs are confirmed as MLs with invasiveness; however, some benign PSNs could disappear spontaneously (16). In terms of management, BLs and MLs are entirely different (17). Therefore, it is important to differentiate benign from malignant PSNs. This study investigated the clinical data of patients and CT features of benign and malignant PSNs, especially the differences of the solid components. It was found that patients with MLs were more likely to be older and non-smokers, and BLs and MLs significantly differed in the CT features of the whole nodule, internal solid component, and GGO. In summary, well-defined border and lobulation of the nodule and irregular shape and scattered distribution of the internal solid components were significant indicators of malignant PSNs.

Most previous studies (18-21) mainly investigated the overall CT features of benign and malignant GGNs and found significant differences in lesion size, border, lobulation spiculation, bubble lucency, air bronchogram, vascular convergence sign, and pleural indentation between BLs and MLs. In addition, some studies (11,12) investigated the overall CT features of BLs and MLs appearing as PSNs. Oh et al. (11) analyzed 86 histologically confirmed or transient PSNs (including 29 malignant and 57 transient 
Table 3 The CT findings of solid components within benign and malignant PSNs

\begin{tabular}{|c|c|c|c|}
\hline CT features & Patients with benign PSNs $(n=44)$ & Patients with malignant PSNs $(n=75)$ & $P$ value \\
\hline Solid component ratio (\%) & $11.3(1.0-28.8)$ & $15.5(2.8-47.1)$ & $0.015^{\dagger}$ \\
\hline Density of Solid component (HU) & $-160(-600$ to 91$)$ & $-80(-495$ to 75$)$ & $0.155^{\dagger}$ \\
\hline Solid component number & & & $<0.001^{\star *}$ \\
\hline Multiple & $3(6.8)$ & $41(54.7)$ & \\
\hline Solid component shape & & & $<0.001^{\star *}$ \\
\hline Round & $25(56.8)$ & 7 (9.3) & \\
\hline Irregular & $19(43.2)$ & $68(90.7)$ & \\
\hline III-defined & $38(86.4)$ & $40(53.3)$ & \\
\hline $\begin{array}{l}\text { Margin of well-defined solid } \\
\text { component }\end{array}$ & & & $0.003^{\star \star \star}$ \\
\hline Smooth & $6(100.0)$ & $11(31.4)$ & \\
\hline Coarse & $0(0.0)$ & $24(68.6)$ & \\
\hline Solid component distribution & & & $<0.001^{\star \star}$ \\
\hline Concentrated & $42(95.5)$ & $37(49.3)$ & \\
\hline Scattered & $2(4.5)$ & $38(50.7)$ & \\
\hline
\end{tabular}

Data are presented as $\mathrm{n}(\%)$ or median (range). ${ }^{* *}$, calculated with the Pearson $\chi^{2}$ test; ${ }^{* *}$, calculated with the Fisher exact test; ${ }^{\dagger}$, MannWhitney U test. CT, computed tomography; PSN, part-solid nodule.

lesions), and found that MLs were more likely to be larger and have spiculation than BLs. Yang et al. (12) reported that larger size, well-defined border, and spiculation had higher predictive value for malignant PSNs, and the AUC for nodule size was 0.652 , which was slightly lower than our result (AUC: 0.734, cut-off value: $12.5 \mathrm{~mm}$ ). Although there are some differences among these studies (due to different inclusion criteria), most of the present findings are consistent with previous reports.

Compared with pure GGNs, the solid components in PSNs make them more characteristic, and therefore, deserve to be meticulously analyzed, which may provide additional findings for the differential diagnosis of BLs and MLs. Previous studies $(12,13,19,22)$ only investigated solid components' size or/and CT value. Yang et al. (12) found that MLs were more likely to have a larger solid component with a higher CT value than BLs, but their morphological manifestations were not well studied. The present study conducted a comprehensive analysis of solid components, and revealed that PSNs with larger, irregular, multiple, scattered, and eccentric solid components had a higher probability of malignancy, while BLs usually had single, round, and ill-defined solid components. However, there was no significant difference in density between MLs and BLs. In addition to the size and CT value of solid components, the current study identified more 

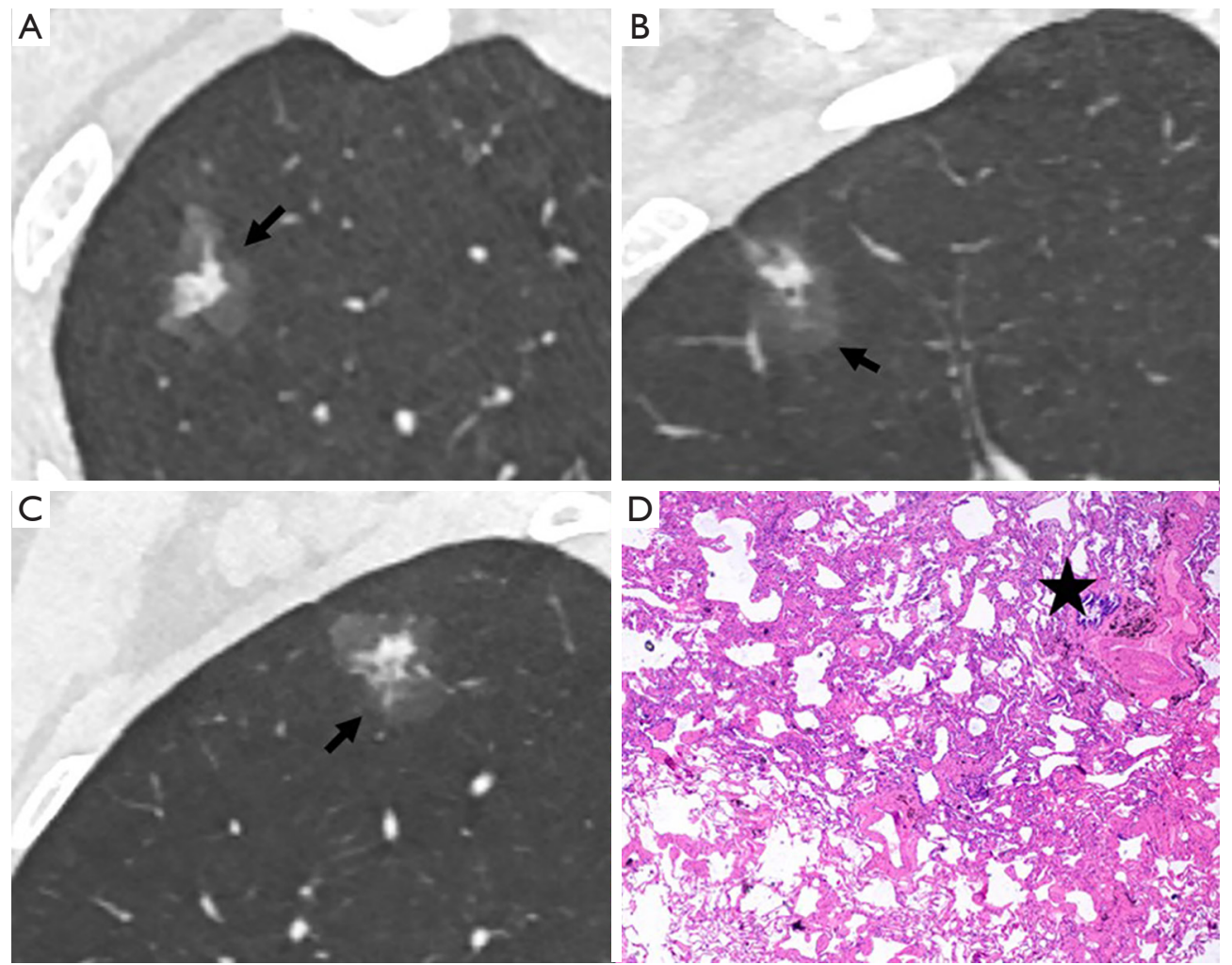

Figure 3 A 50-year-old woman with an invasive adenocarcinoma. (A-C) Axial (A), coronal (B), and sagittal (C) CT images show a 17-mm irregular and well-defined PSN (arrows) in the right upper lobe. The internal solid component is eccentric, irregular, and well-defined, and the peripheral GGO is homogeneous. (D) Photomicrograph (hematoxylin-eosin stain, $\times 100$ ) of the PSN reveals adenocarcinoma with invasive foci (asterisk). CT, computed tomography; GGO, ground-glass opacity; PSN, part-solid nodule.

characteristics with predictive value, which could provide new information for a more accurate diagnosis. Therefore, solid components are an important part of PSNs and are highly significant for diagnosis.

Pathologically, the solid components in MLs have been confirmed as tumor invasion with or without alveolar collapse and/or fibroblastic proliferation (23). Different cell differentiation degrees and growth rates result in irregular shapes of solid components, and the multiple scattered solid components may be related to multiple origins of invasions. As for benign PSNs, most are inflammatory lesions. The internal solid components usually represent a large number of inflammatory cells, and GGOs represent exudates and fibrotic changes (1). This indicates that inflammation in solid components is more severe than that in peripheral GGOs, and peripheral GGOs might be secondary to central inflammation. Thus, in benign PSNs, the border of the solid component and its peripheral GGO are usually illdefined.

Our study had two limitations that should be noted. Firstly, the sample size of benign PSNs was small. Therefore, more cases are needed in future studies to confirm this generalization. Secondly, this is a retrospective study; thus, the samples were mainly selected from patients who underwent surgical resection. This study could not provide the precise number of PSNs that were being followed or lost to follow-up and should be excluded.

\section{Conclusions}

The CT features of benign and malignant PSNs were markedly different. Well-defined and lobulated PSNs with irregular, multiple, and scattered solid components are highly likely to be malignant. In contrast, PSNs with sole, 

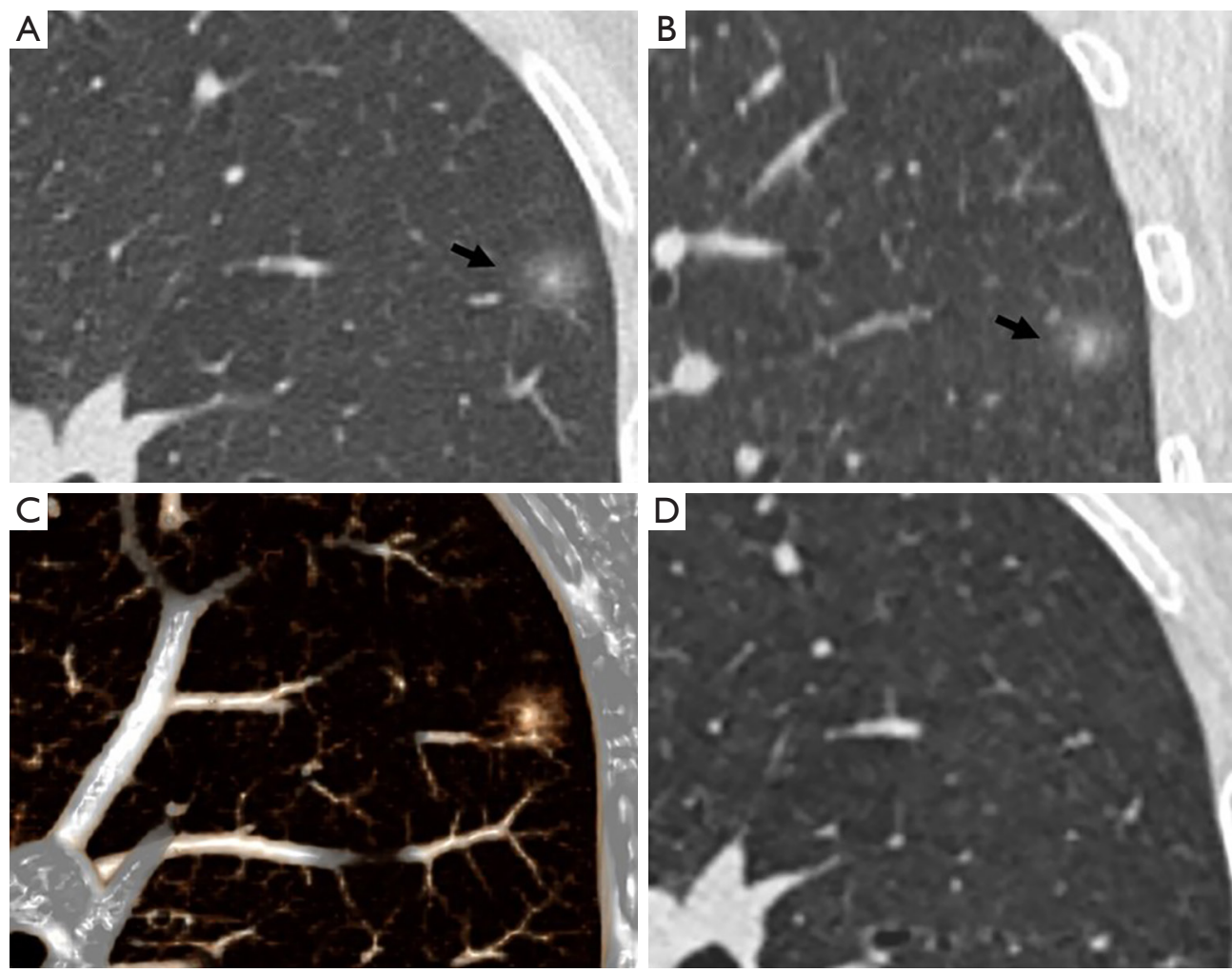

Figure 4 A 63-year-old woman with a transient PSN in the left upper lobe. (A,B) Axial (A) and sagittal (B) CT images show an 8-mm round PSN (arrows). The internal high-attenuation zone is centric, round, and ill-defined, and the peripheral GGO is ill-defined. (C) VR image. (D) At the follow-up CT performed 3 months later, the PSN had disappeared. CT, computed tomography; GGO, ground-glass opacity; PSN, part-solid nodule; VR, volume rendering.

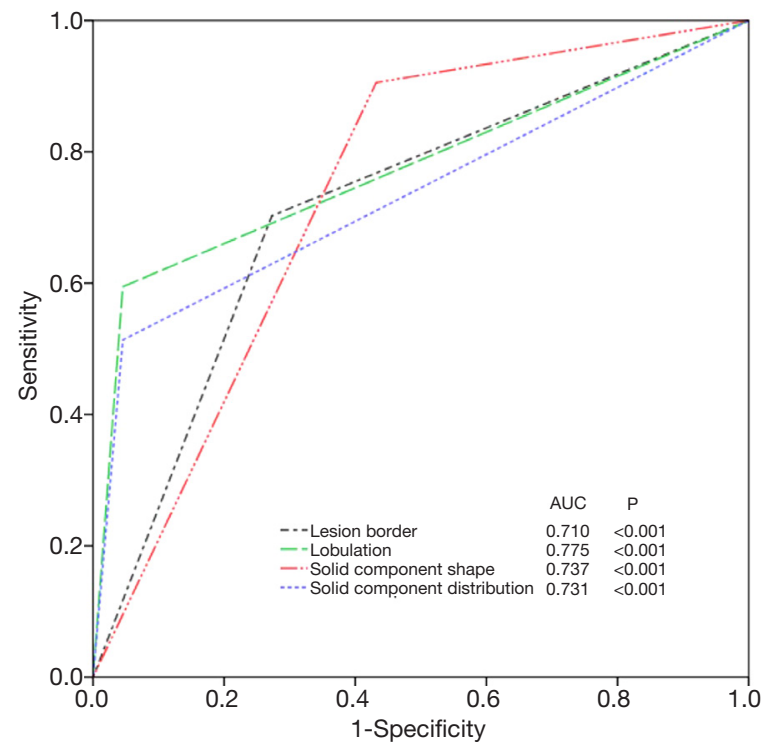

Figure 5 Receiver operating characteristic curve for lesion border, lobulation, solid component shape, and distribution in part-solid nodules. AUC, area under the curve. round, and blurred solid components indicate BLs.

\section{Acknowledgments}

Funding: This study was supported by the National Natural Science Foundation of China (81601545) and the Chongqing Health and Family Planning Commission Foundation of China (2016MSXM018).

\section{Footnote}

Conflicts of Interest: All authors have completed the ICMJE uniform disclosure form (available at https://dx.doi. org/10.21037/qims-21-145). Dr. ZGC reports this study was supported by the National Natural Science Foundation of China (81601545) and the Chongqing Health and Family Planning Commission Foundation of China (2016MSXM018). The other authors have no conflicts of interest to declare. Ethical Statement: The authors are accountable for all 
aspects of the work in ensuring that questions related to the accuracy or integrity of any part of the work are appropriately investigated and resolved. The retrospective study was conducted in accordance with the Declaration of Helsinki (as revised in 2013) and was approved by the ethics committee of the First Affiliated Hospital of Chongqing Medical University. The requirement for written informed consent was waived due to the retrospective nature of the study.

Open Access Statement: This is an Open Access article distributed in accordance with the Creative Commons Attribution-NonCommercial-NoDerivs 4.0 International License (CC BY-NC-ND 4.0), which permits the noncommercial replication and distribution of the article with the strict proviso that no changes or edits are made and the original work is properly cited (including links to both the formal publication through the relevant DOI and the license). See: https://creativecommons.org/licenses/by-nc-nd/4.0/.

\section{References}

1. Park CM, Goo JM, Lee HJ, Lee CH, Chun EJ, Im JG. Nodular ground-glass opacity at thin-section CT: histologic correlation and evaluation of change at followup. Radiographics 2007;27:391-408.

2. Mao R, She Y, Zhu E, Chen D, Dai C, Wu C, Xie H, Zhu H, Fei K, Chen C. A Proposal for Restaging of Invasive Lung Adenocarcinoma Manifesting as Pure Ground Glass Opacity. Ann Thorac Surg 2019;107:1523-31.

3. She Y, Zhao L, Dai C, Ren Y, Zha J, Xie H, Jiang S, Shi J, Shi S, Shi W, Yu B, Jiang G, Fei K, Chen Y, Chen C. Preoperative nomogram for identifying invasive pulmonary adenocarcinoma in patients with pure ground-glass nodule: A multi-institutional study. Oncotarget 2017;8:17229-38.

4. Kim HY, Shim YM, Lee KS, Han J, Yi CA, Kim YK. Persistent pulmonary nodular ground-glass opacity at thin-section CT: histopathologic comparisons. Radiology 2007;245:267-75.

5. Naidich DP, Bankier AA, MacMahon H, Schaefer-Prokop CM, Pistolesi M, Goo JM, Macchiarini P, Crapo JD, Herold CJ, Austin JH, Travis WD. Recommendations for the management of subsolid pulmonary nodules detected at CT: a statement from the Fleischner Society. Radiology 2013;266:304-17.

6. Lee HJ, Goo JM, Lee CH, Yoo CG, Kim YT, Im JG. Nodular ground-glass opacities on thin-section CT: size change during follow-up and pathological results. Korean
J Radiol 2007;8:22-31.

7. Henschke CI, Yankelevitz DF, Mirtcheva R, McGuinness G, McCauley D, Miettinen OS; ELCAP Group. CT screening for lung cancer: frequency and significance of part-solid and nonsolid nodules. AJR Am J Roentgenol 2002;178:1053-7.

8. Ye T, Deng L, Wang S, Xiang J, Zhang Y, Hu H, Sun Y, Li Y, Shen L, Xie L, Gu W, Zhao Y, Fu F, Peng W, Chen H. Lung Adenocarcinomas Manifesting as Radiological PartSolid Nodules Define a Special Clinical Subtype. J Thorac Oncol 2019;14:617-27.

9. Nakajima R, Yokose T, Kakinuma R, Nagai K, Nishiwaki Y, Ochiai A. Localized pure ground-glass opacity on highresolution CT: histologic characteristics. J Comput Assist Tomogr 2002;26:323-9.

10. Yamada N, Kusumoto M, Maeshima A, Suzuki K, Matsuno Y. Correlation of the solid part on high-resolution computed tomography with pathological scar in small lung adenocarcinomas. Jpn J Clin Oncol 2007;37:913-7.

11. Oh JY, Kwon SY, Yoon HI, Lee SM, Yim JJ, Lee JH, Yoo CG, Kim YW, Han SK, Shim YS, Kim TJ, Lee KW, Chung JH, Jheon SH, Sung SW, Lee CT. Clinical significance of a solitary ground-glass opacity (GGO) lesion of the lung detected by chest CT. Lung Cancer 2007;55:67-73.

12. Yang W, Sun Y, Fang W, Qian F, Ye J, Chen Q, Jiang Y, Yu K, Han B. High-resolution Computed Tomography Features Distinguishing Benign and Malignant Lesions Manifesting as Persistent Solitary Subsolid Nodules. Clin Lung Cancer 2018;19:e75-83.

13. Lee SM, Park CM, Goo JM, Lee CH, Lee HJ, Kim KG, Kang MJ, Lee IS. Transient part-solid nodules detected at screening thin-section CT for lung cancer: comparison with persistent part-solid nodules. Radiology 2010;255:242-51.

14. Zhang BW, Zhang Y, Ye JD, Qiang JW. Use of relative CT values to evaluate the invasiveness of pulmonary subsolid nodules in patients with emphysema. Quant Imaging Med Surg 2021;11:204-14.

15. Travis WD, Brambilla E, Burke AP, Marx A, Nicholson AG. Introduction to The 2015 World Health Organization Classification of Tumors of the Lung, Pleura, Thymus, and Heart. J Thorac Oncol 2015;10:1240-2.

16. Choi WS, Park CM, Song YS, Lee SM, Wi JY, Goo JM. Transient subsolid nodules in patients with extrapulmonary malignancies: their frequency and differential features. Acta Radiol 2015;56:428-37.

17. Pedersen JH, Saghir Z, Wille MM, Thomsen LH, Skov 
BG, Ashraf H. Ground-Glass Opacity Lung Nodules in the Era of Lung Cancer CT Screening: Radiology, Pathology, and Clinical Management. Oncology (Williston Park) 2016;30:266-74.

18. Fan L, Liu SY, Li QC, Yu H, Xiao XS. Multidetector CT features of pulmonary focal ground-glass opacity: differences between benign and malignant. Br J Radiol 2012;85:897-904.

19. Hu H, Wang Q, Tang H, Xiong L, Lin Q. Multislice computed tomography characteristics of solitary pulmonary ground-glass nodules: Differences between malignant and benign. Thorac Cancer 2016;7:80-7 .

20. Gao F, Sun Y, Zhang G, Zheng X, Li M, Hua Y. CT characterization of different pathological types of subcentimeter pulmonary ground-glass nodular lesions. $\mathrm{Br}$ J Radiol 2019;92:20180204.

Cite this article as: $\mathrm{Li} \mathrm{WJ}, \mathrm{Lv} F J$, Tan $\mathrm{YW}, \mathrm{Fu} \mathrm{BJ}$, Chu ZG. Benign and malignant pulmonary part-solid nodules: differentiation via thin-section computed tomography. Quant Imaging Med Surg 2022;12(1):699-710. doi: 10.21037/qims21-145
21. Qiu ZX, Cheng Y, Liu D, Wang WY, Wu X, Wu WL, Li WM. Clinical, pathological, and radiological characteristics of solitary ground-glass opacity lung nodules on highresolution computed tomography. Ther Clin Risk Manag 2016;12:1445-53.

22. Chen ML, Li XT, Wei YY, Qi LP, Sun YS. Can spectral computed tomography imaging improve the differentiation between malignant and benign pulmonary lesions manifesting as solitary pure ground glass, mixed ground glass, and solid nodules? Thorac Cancer 2019;10:234-42.

23. Lee HJ, Lee CH, Jeong YJ, Chung DH, Goo JM, Park CM, Austin JH. IASLC/ATS/ERS International Multidisciplinary Classification of Lung Adenocarcinoma: novel concepts and radiologic implications. J Thorac Imaging 2012;27:340-53. 